\title{
Comunidad de Paz: Una paz 'otra' en San José de Apartadó-Colombia ${ }^{2}$
}

\author{
Community of Peace: An 'other' Peace in San José de Apartadó, Colombia \\ Comunidade de Paz: Uma paz 'outra' em San José de Apartadó, Colombia
}

\begin{abstract}
Resumen
Este artículo de investigación problematiza el concepto de paz a partir de su concepción y práctica en la Comunidad de Paz de San José de Apartadó, Colombia. Se basa en 16 meses de investigación etnográfica en Colombia entre 2011 y 2016, incluyendo 49 entrevistas con miembros de la Comunidad de Paz de San José de Apartadó y otras organizaciones. Al definir y crear la paz, como al negarse a colaborar con los grupos armados y la construcción de comunidad a través del trabajo colectivo esta experiencia campesina encarna una perspectiva activa y empoderada de la paz en lugar de depender de lo que deberían hacer el Estado y los grupos armados. En conversación con los nuevos subcampos de estudios de paz en geografía, y las investigaciones sobre Trans-rational peace, se aboga por una conceptualización de paz como la construcción de vida digna en las redes de solidaridad de las comunidades en resistencia que contrarresta las violencias interseccionales de la crisis ecológica de la modernidad.
\end{abstract}

Palabras clave: paz, modernidad, relacionalidad, comunidad de paz, geografías de paz.

\section{Recibido: 21 de noviembre, evaluado: 8 de diciembre, aprobado: 9 de diciembre}

1 Doctor en Geografía de la Universidad de Carolina del Norte, Chapel Hill, Estados Unidos. Investigador y docente del Programa de Estudios de Paz y Ciudadanía de la Corporación Universitaria Minuto de Dios. Correo electrónico: christopher.courtheyn@uniminuto.edu

2 Este artículo de investigación, presenta algunos de los resultados de la tesis para optar por el título de Doctorado, titulada: Memory is the strength of our resistance: A critical performance geography of peace, memory, territory, and politics in the San Jose Peace Community, Colombia. 


\begin{abstract}
This article problematizes the concept of peace by exploring the conception and practice of peace in the peasant Peace Community of San José de Apartadó, Colombia. This scientific research article is base don 16 months of ethnographic research in Colombia from 2011 to 2016, including 49 interviews with members of the San José de Apartadó Peace Community and other organizations. Given their definition and creation of peace as the refusal to collaborate with the armed groups and as the construction of community through community work, this experience embodies an active and empowered perspective on peace, rather tan depending upon what states or armed actors should do. In conversation with new subfields of peace studies in geography and transrational peace research, the article calls for a conceptualization of peace as the construction of dignified life through the solidarity networks of communities in resistance, which counter the intersectional violences of the ecological crisis of modernity.
\end{abstract}

Keywords: Peace, Modernity, Relationality, Peace Communities, Peace Geographies.

\title{
Resumo
}

Este artigo problematiza o conceito de paz a partir de sua concepção e prática na Comunidade de Paz de San José de Apartadó, Colômbia. Este artigo de investigação científica baseia-se em 16 meses de investigação etnográfica em Colômbia entre 2011 e 2016, incluindo 49 entrevistas com membros da Comunidade de Paz de San José de Apartadó e outras organizações. Ao definir e criar a paz, como ao se negar a colaborar com os grupos armados e a construção de comunidade através do trabalho coletivo esta experiência camponesa encarna uma perspectiva ativa e empoderada da paz em lugar de depender do que deveriam fazer o Estado e os grupos armados. Em conversa com os novos subcampos de estudos de paz em geografia, e as investigações sobre Trans-rational peace, se defende por uma conceitualização de paz como a construção de vida digna nas redes de solidariedade das comunidades em resistência que neutraliza as violências inter seccionais da crise ecológica da modernidade.

Palavras-chave: paz, modernidade, relacionalidade, comunidade de paz, geografias da paz. 


\section{Introducción}

En el mundo de hoy, permeado de los enfrentamientos militares, la pobreza, la violación y la degradación ambiental, ¿es posible la paz? Por lo general se piensa en la paz como una condición utópica de 'tranquilidad' o 'no guerra', tal perspectiva se ve reflejada en el actual 'proceso de paz' entre el Gobierno Colombiano y las Fuerzas Armadas Revolucionarias de Colombia (FARC) que busca la dejación de armas de esa guerrilla insurgente y su transformación en un partido político. Después de cinco años de negociaciones, en 2016 las partes llegaron a un acuerdo final para poner fin a esta confrontación armada que duró más de cincuenta años y causó al menos 220.000 asesinatos (Grupo de Memoria Histórica, 2013).

¿Cómo concebimos la paz más allá de esta oposición simplista a la guerra? Varios teóricos críticos han demostrado que lo que comúnmente se llama 'paz' frecuentemente no es lo contrario de la guerra en absoluto. Más bien, puede reflejar la victoria de un grupo armado sobre otro (por ej., Dalby, 2014 y Foucault, 2003); asimismo, la represión de la protesta en contra de la injusticia (Ross, 2011), o la presencia arraigada del patriarcado, el racismo y la desigualdad (Daley, 2014; Darling, 2014; Federici, 2010).

Este artículo analiza la creación de una paz 'otra' desde la resistencia campesina. En 1997 un grupo de mil campesinos se declaró como 'comunidad de paz’ en el corregimiento de San José de Apartadó, en el Urabá antioqueño; una región limítrofe con Panamá. La región de Urabá se encuentra entre las zonas más devastadas por la guerra en Colombia, donde los intereses estatales, empresariales e insurgentes compiten para controlar el tráfico de drogas y armas desde y hacia América del Norte, así como las tierras fértiles de la región, las plantaciones de banano, y las reservas de agua y minerales. Sin embargo, a pesar del asesinato de más de doscientas personas en San José por los paramilitares, el Ejército Nacional y las guerrillas, con los cuales sus campesinos se niegan a colaborar (Giraldo, 2010), la Comunidad de Paz se ha expandido de una a once veredas, durante los últimos veinte años.
En lugar de nociones pasivas de 'no guerra' o 'tranquilidad', los miembros de la Comunidad de Paz definen la paz activamente como: a) negarse a colaborar con los grupos armados y b) la construcción de comunidad a través del trabajo colectivo. Así practican esa paz, cultivando con fines de comercialización y para el pancoger en grupos de trabajo y en tierras comunes. Además, toman decisiones colectivamente en asambleas comunitarias, conmemoran a las víctimas con peregrinaciones a sitios de masacre y pintando piedras con los nombres de las víctimas, y participan en redes nacionales e internacionales con otras organizaciones de derechos humanos y comunidades campesinas indígenas y afrodescendientes.

La paz se asocia frecuentemente a la pacificación militar o a la diplomacia estatal, visión que se ha extendido hegemónicamente por todo el mundo con la globalización de esa paz 'modernaliberal' a través del colonialismo y el desarrollo (Daley, 2014; Gebrewold, 2011), sin embargo, la Comunidad de Paz muestra que la paz es un proceso que puede ser producido por actores no estatales, a través de prácticas cotidianas para sembrar condiciones dignas de vida.

Nutrido por esta experiencia campesina de paz y por los recientes avances en la teoría de la paz en dos subcampos pioneros de estudios de paz - geografías de la paz y la investigación en Trans-rational peace-, este artículo problematiza el concepto de paz; describe como este grupo de campesinos sujeto a la violencia política re-construye espacios comunitarios para la construcción de la paz y aboga por una conceptualización de paz trasrelacional radical, entendida como dignidad ecológica entre los miembros de la comunidad, con otras comunidades y con la tierra.

\section{Descripción metodológica: Geografía crítica y performativa}

La auto-gestión de la paz por los movimientos sociales es una práctica encarnada y espacial, por la cual su comprensión requiere una metodología comprometida. Mi metodología es lo que llamo geografía crítica y performativa, la cual integra la 
teoría crítica (análisis de sistemas-mundo; geopolíticas feministas; teoría crítica de la raza) y la investigación colaborativa (con énfasis en métodos performativos) $)^{3}$. Realicé 16 meses de investigación etnográfica en Colombia entre 2011 y 2016, incluyendo 49 entrevistas con miembros de la Comunidad de Paz de San José de Apartadó, miembros de otras organizaciones sociales y oficiales de la fuerza pública. Este estudio científico se basa en mi relación histórica en San José, donde trabajé desde 2008-2010 como acompañante internacional, una estrategia de seguridad donde los defensores de los derechos humanos amenazados invitan a 'guardaespaldas no armados' (Mahony \& Eguren, 1997), los cuales también difunden las experiencias de las comunidades de paz a nivel global (Koopman, 2011a).

Mi trabajo es critico en la medida que, al reconocer mi lugar de enunciación como geógrafo, resulto ser inevitablemente un participante en la construcción de formas particulares de paz a través de mi presencia y publicaciones (Vasudevan, 2012). Tomo en serio estas apuestas poniendo de relieve las voces de mis interlocutores y uniendo mi análisis con las teorías críticas de la paz necesarias para comprender plenamente la praxis de la Comunidad de Paz. Además, esta es una geografía performativa porque reconozco a mi cuerpo como investigador que marcha en una peregrinación campesina y que representa la solidaridad internacional, tanto para la comunidad como para los actores armados. También abordo la entrevista no como una extracción de datos sino como un espacio performativo de la producción del conocimiento, donde la conversación recíproca permite reflexión continua, además de la crítica y la construcción de la teoría (Madison, 2012; Pollock, 2005). Finalmente, presento mi etnografía con representaciones performativas que en este artículo se verá evidenciado en fragmentos de entrevistas en los que se incluyen también mis propias preguntas, a partir de las cuales se representa el diálogo.

\section{Re-conceptualizando la paz en medio de la crisis de la modernidad}

Durante décadas, los conceptos de Johan Galtung $(1964,1996)$ de 'paz negativa' (ausencia de guerra abierta) y 'paz positiva' (la ausencia de violencia directa, estructural y cultural a través de la integración de la sociedad humana) han dominado la teoría de la paz. Estos conceptos canónicos aportan una diferenciación clave entre la ausencia de violencia directa y una paz integral de justicia social. Sin embargo, la paz positiva sigue siendo una concepción vaga (Koopman, 2011b) y puede limitarnos a marcos modernistas (Mitchell, 2012).

Ahora bien, dos nuevos campos de estudio de paz, la Trans-rational peace (Dietrich, 2012 \& Dietrich, Echavarría, Esteva, Ingruber, \& Koppensteiner, 2011) y las geografías de paz (McConnell, Megoran, \& Williams, 2014), han emergido en la última década para problematizar el concepto de la paz. Explorando una variedad de conceptos y prácticas de paz en una multitud de culturas y lugares del mundo, han iluminado una 'pluralidad de las paces'. Estos imaginarios van desde el fridr vikingo que entiende la paz como la fertilidad (Jacobsen, 2011), hasta la salaam islámica con énfasis en la hospitalidad (Dietrich, 2012; Fasheh, 2011), o la armonía cosmo-ecológica thaq de los quechua (Rengifo, 2011).

Wolfgang Dietrich (2012) categorizó estas many peaces o multiplicidad de las paces, en cuatro familias: 'la paz energética' (paz como la fertilidad o la armonía entre los seres humanos, la naturaleza y el cosmos); 'paz moral' (paz normativa a través de la justicia social, o la salvación a través de la creencia en un 'Dios Único'); 'paz moderna' (la seguridad garantizada por el estado-nación), y 'paz posmoderna' (paz a través de la verdad de la multiplicidad de las paces). Pero Dietrich también acuñó su propia noción, como un intento de articular los

3 El desarrollo de esta metodología geografía critica y performativa, se realiza con mayor profundidad en la tesis doctoral (Courtheyn, 2016a).

Polisemia No. 22, 55 - 72. Comunidad de Paz: Una paz 'otra' en San José de Apartadó-Colombia. Bogotá, ISSN: 1900-4648. Julio - diciembre de 2016 
elementos de las otras familias en un marco holístico para teorizar y practicar la paz que integra tanto la paz interior-personal como la paz socioculturalcomunitaria. Sin embargo, se evidencia la falta de integración de los aportes de esas literaturas en el libro destacado Paz en Colombia-publicado por Clacso (Rueda, Alvarado, \& Gentili, 2016)-, lo cual podría significar que los estudios de paz en Colombia todavía no se han nutrido de esas literaturas innovadoras, a pesar de las contribuciones que su exploración de esas many peaces podrá aportar a la teoría y la construcción de la paz.

Conjuntamente, las geografías de paz y la investigación en Trans-rational peace contribuyen con varios aportes a la teoría de paz. Primero, destacan que la paz sirve como un discurso político (Megoran, Williams, \& McConnell, 2014), el cual puede funcionar para reprimir (Ross, 2011) o emancipar (McConnell, 2014). Segundo, hacen una crítica profunda a la paz moderna o 'liberal', construida a partir de la 'seguridad' para los ciudadanos, garantizada o impuesta por el Estadonación, la cual reproduce la dominación del Estado sobre sus súbditos y los no ciudadanos, el hombre sobre la mujer, y las empresas capitalistas sobre sus trabajadores y la biosfera (Daley, 2014; Dietrich, 2002, 2012).

Ahora bien, al explorar la diversidad de ideas sobre la paz, estas literaturas demuestran que la paz no tendrá que ser entendida como una condición utópica del futuro, como se pretende desde la noción de paz moderna, que la busca sin alcanzarla a través de operaciones militares y tratados entre Estados, sino que la paz es siempre un proceso, la cual se puede producir a través de las prácticas cotidianas (Loyd, 2012; Williams, 2013). Por ejemplo, formas de 'paz moral' desde la hospitalidad, como la salaam islámica -Como ya se ha mencionado-, el shalom judío (Fasheh, 2011) o el t'ummu del pueblo Kambaata en Etiopía (Gebrewold, 2011), que sostiene que la paz se puede construir entre vecinos y desconocidos siguiendo normas de cuidado interpersonal. Además, la 'paz energética', enfocada en armonía ecológica entre los seres humanos, la naturaleza y los cosmos, pone énfasis en las relaciones más allá de la humanidad que son necesarias para la sustentabilidad y la dignificación de la vida. Además, el argumento que el conflicto es fundamental para la paz (Muñoz, 2001) - en que las tensiones producidas por las diferencias nutren la creatividad para construir nuevas formas de vida (Dietrich, 2011) — nos ayuda a distinguir entre el conflicto y la guerra o la violencia, esta última que he podido conceptualizar como la resolución de conflictos a partir de la violación de una o más de las partes.

Explorando esta amplia gama de 'paces', incluyendo, pero yendo más allá de la 'paz moderna', las geografías de paz y la investigación en Transrational peace ilustran los proyectos político-socioecológicos divergentes, implícitos en las distintas formas de paz. No obstante, si se considera la crisis ecológica actual alrededor del mundo, enraizada en violencias interseccionales (Martínez, 2013), la medida en que estas diversas ideas y prácticas de paz contribuyen a transformar o, de hecho, exacerban esta crisis, es bastante variada. Por eso, sostengo que iluminar una 'pluralidad de las paces' es insuficiente. No todas las formas necesariamente contribuyen a "relaciones sostenibles y justas", para usar la definición operativa de paz de Megoran (2011, p. 182). Por ejemplo, al describir los viajes trasnacionales de un cantante de Kirguistán a Israel como una geografía de paz, aún cuando ese cantante es un millonario que apoya la ocupación israelí de territorio palestino, Megoran admite que "la paz para un grupo de personas puede ser mala noticia para otro" (2014, p. 225). De hecho, lo que se llama 'paz' frecuentemente refleja la victoria de un grupo armado sobre otro (Dalby, 2014; Foucault, 2003), la represión de la protesta en contra de la injusticia (Ross, 2011), o las jerarquías arraigadas de explotación patriarcal, racista y clasista (Daley, 2014; Federici, 2010; Loyd, 2012), las cuales yo argumento que no se deben considerarse 'paz' en absoluto.

Sostengo que estos nuevos campos de estudio de paz todavía carecen de un marco normativo que evalúe la 'pluralidad de las paces'. Como calificador referencial para la diversidad de teorías y proyectos 
de paz propongo la paz transrelacional radical: dignidad ecológica a través de y por las redes de solidaridad entre comunidades. Mientras Dietrich (2014) construye su concepción de paz integral desde la posracionalidad, encuentro más posibilidades para una paz integral ecológica a partir de la noción de relacionalidad. Este concepto hace hincapié en la necesidad de 'ontologías relacionales' (De la Cadena, 2015; Escobar, 2014) que contrarresten los dualismos jerárquicos de la modernidad, incluyendo el hombre sobre la naturaleza, el hombre sobre la mujer, el blanco sobre el negro e indígena, y la burguesía capitalista sobre los trabajadores. Con respecto a estos dualismos, Carlos Eduardo Martínez Hincapié (2010) observa:

Esta cultura hegemónica necesita construir límites y fronteras para, desde allí, definir las identidades individuales y colectivas. Nuestras mentes están plagadas de muros que nos separan de los demás seres vivos como condición de reconocimiento de nuestra propia especie. Hemos construido el concepto de nación desde la delimitación del territorio que nos separa de los otros pueblos. Hemos levantado una frontera entre los roles que nos identifican como hombres o mujeres para definir nuestra identidad sexual. Delimitamos nuestras verdades religiosas para poder sentir a Dios como una posesión exclusiva. Hemos separado la racionalidad y la sensibilidad, el cuerpo y el alma hasta llegar a construir identidades esquizofrénicas. Y creemos entender mejor el mundo haciendo de las diferentes ciencias compartimientos estancos claramente diferenciados. (pp. 22-23).

Identifico a la modernidad como la cultura sociopolítica que surgió a través de la colonización europea de las Américas. Este sistema mundial capitalista celebra el individualismo, la igualdad, la democracia liberal, la soberanía del Estado-nación, la propiedad privada, el universalismo y el progreso. Pero siempre ha sido estructurado por relaciones desiguales de explotación de centro-periferia (Wallerstein, 2004) y por dualismos jerárquicos como civilización-barbarismo y razón-emoción (Escobar [en prensa], \& Gómez, 2016). En otras palabras, la modernidad se refiere tanto a una época (Dussel, 2000) como a una 'actitud' (Dietrich, 2012; Foucault, 1984).
Del cambio climático a la violencia sistemática en contra de las poblaciones racializadas, "[...] nos enfrentamos a problemas modernos para los que no hay soluciones modernas" (Santos, 2002, p. 13). Por lo tanto, abordar esta crisis requiere rupturas radicales con las estructuras institucionales y la reproducción social modernas. Empleo el término 'radical' para señalar acciones y perspectivas que confrontan 'la raíz' de un problema (Madison, 2010) y posibilitan otros sistemas sociopolíticos (Wallerstein, 2004).

Cabe señalar que una alternativa radical no es una mera utopía. Se puede observar en ciertos movimientos sociales actuales, como las comunidades autónomas en resistencia en América Latina, los cuales se ha identificado como una 'otra política' (Denis, 2012; Grupo Acontecimiento, 2012) de 'emancipación' de la dominación moderna (Ceceña, 2012; Gutiérrez, 2012; Useche, 2016). Reflejando la 'autonomía relacional' (Ulloa, 2012) en lugar del aislacionismo; estas comunidades luchan por la soberanía alimentaria a través de redes solidarias contra la destrucción ecológica del extractivismo y la violencia capitalista. Esto coincide con la insistencia de que la reproducción social o la 'paz' en un lugar no debe venir a expensas de la vida y la dignidad en otro lugar (Federici, 2010). Entonces, la paz transrelacional radical cuestiona el aislamiento de contextos y aboga por una comprensión más matizada de la forma en que los múltiples 'locales' nutren o socavan la dignidad ecológica en un mundo globalizado.

La Comunidad de Paz de San José de Apartadó en Colombia ejemplifica la conexión entre esta otra política y la paz. Fundada en el 1997 en el Urabá antioqueño, este grupo de mil campesinos ha resistido por dos décadas el desplazamiento forzado de sus tierras y la cooptación de paramilitares, el Ejército Nacional y las guerrillas. A pesar de una serie de desplazamientos forzados y el asesinato de más de doscientos campesinos desde el 1997 por los actores armados (Giraldo, 2010), la mayoría por parte de las fuerzas estatales y paramilitares, la Comunidad sigue resistiendo en once veredas. Su proyecto de paz se hace realidad a través de una 
variedad de prácticas. Toman decisiones colectivamente en reuniones de aldeas y asambleas comunitarias. Cultivan alimentos en grupos de trabajo, incluyendo cacao, frijoles, maíz, aguacate y caña de azúcar. Se comprometen a trabajar colectivamente un día a la semana para construir casas y reparar senderos, en lo que ellos han llamado Comunitario. Conmemoran las masacres de sus miembros con peregrinaciones a los sitios, donde narran los acontecimientos y pintan piedras con los nombres de las víctimas. Y participan en redes de solidaridad con organizaciones de derechos humanos y otras comunidades, como la Universidad Campesina de la Resistencia, en la que grupos de descendencia indígena y afro ${ }^{4}$ comparten y se producen conocimientos para la construcción de dignidad, y soberanía alimentaria en sus comunidades. Todas estas prácticas inspiran la noción de paz radical trasrelacional, que será la tesis de este trabajo.

Conceptualizar la paz desde una comunidad campesina es oportuno porque como lo expresa Diego Fernando Silva Prada (2012) "La capacidad de organización de las comunidades campesinas ha demostrado que la resistencia tiene posibilidades de realización histórica, incluso en contextos altamente desfavorables, y que es posible la concreción de alternativas sociales y políticas" (p. 166), especialmente respecto a la paz. Me dirijo, entonces, a explorar las visiones de paz que tienen los campesinos de la Comunidad de Paz.

\section{La paz según la Comunidad de Paz}

¿Qué es la paz para la Comunidad de Paz de San José de Apartadó, teniendo en cuenta que se definen usando este mismo concepto? Inicio con un fragmento de una entrevista realizada en San José, la primera en que explícitamente indagué sobre las concepciones de la paz en esa Comunidad, a través de mi pregunta inicial:

Entonces, como alguien que ha participado en este proceso de Comunidad de Paz ¿qué es la paz para usted?

La paz para mí es tener, es tener [...] cómo le digo yo [...] de estar todos juntitos a una misma idea.

\section{Interesante...}

Sí [...] ni el uno bravo, ni uno para aquí, otro para allí, y otro para allá. [...] Que se pueda hacer las cosas [...] [Si] uno se tira para allí, el otro tira para allá, [...] no se compone. Sino tiene todas las cosas descompuestas. [...] Tener todo de acuerdo a una misma idea.

Entonces, ¿la paz es comunidad?

La paz es comunidad.

Y ¿cómo es para ir hacia esa paz? ¿Cuál es el camino? (Fragmento entrevista personal, 2013) .

4 Los habitantes de San José de Apartadó se identifican como campesinos, a los cuales se les suele categorizar dentro del esquema racial como mestizos. Sin embargo, según a) las teorías críiticas de la raza que la definen como la jerarquía social moderna que incluye y excluye ciertos grupos de la humanidad, en lugar de ser una "identidad" (Fanon, 2008; Goldberg, 2009; Wilderson, 2010), b) la historia del colonialismo interno a través de las misiones y la educación estatales-católicas en Antioquia (Steiner, 2000), y c) el reconocimiento de los líderes comunitarios de que "Por naturaleza, somos de sangre indígena, y la llevamos adentro" (apuntes de campo, 2012), sostengo que el identificador "campesino mestizo" oculta el proceso por el cual han sido históricamente "desindigenizados", una observación que le debo a Álvaro Reyes (comunicación personal, 23 de julio de 2015) y De la Cadena (2000). No obstante, estos campesinos siguen siendo racializados, es decir, por su fenotipo y autonomía política son marcados con una posición de sujeto "subhumana" como el "salvaje indígena" que el proyecto colonial-moderno busca exterminar (Rojas, 2002; Wilderson, 2010), dinámica que se manifiesta en la violencia masiva en su contra desde el Estado colombiano, aun después de negarse a colaborarle a la guerrilla. De hecho, en un encuentro de la Universidad Campesina, indígenas y campesinos afirmaron que "todos enfrentamos la misma violencia" (apuntes de campo, 2013). Por lo tanto, mientras que los grupos tienen diferentes identidades y prácticas culturales, la violencia contra los campesinos indígenas y afrodescendientes sugiere una posición social análoga como sujetos racializados. Por lo tanto, me parece imprescindible señalar que la Comunidad de Paz está compuesta en su mayoría por campesinos indígenas (en vez de campesinos "blancos").

5 No se hace alusión al nombre propio de algunas de las personas de la comunidad que participan en esta investigación, por su propia solicitud o por motivos de seguridad. 
La explicación de esta miembro de la Comunidad fue una ruptura con las definiciones de la paz que yo había encontrado hasta entonces. Ella no redujo la paz a una condición material o social, y no mencionó la guerra en absoluto. En cambio, ella enfatizó la unidad y el trabajo colectivo comunitarios. Empecé a ver cómo se podría definir la paz independientemente de la violencia directa. Sólo después de escucharle decir que "la paz es comunidad" me di cuenta que esperaba, inconscientemente, que ella definiera la paz como viviendo sin guerra, como armonía, o cuando la gente tiene sus necesidades materiales básicas satisfechas. Esas últimas visiones correspondían a lo que yo había escuchado de otros colombianos ${ }^{6}$ y leído en la literatura académica sobre la paz: la paz 'negativa' o 'positiva'.

Al definir la paz como "estar todos juntos a una misma idea”, hay un sentido de convivencia utópica y la necesidad de cierta uniformidad entre el grupo. Sin embargo, en la práctica, la diferencia y el conflicto son centrales en el proceso de la Comunidad de Paz. En su documento de fundación, nombran como principios fundamentales tanto el "respeto a la pluralidad" como la 'libertad' de la Comunidad y de los miembros individuales de tomar sus propias decisiones (Comunidad de Paz de San José de Apartadó, 2005). Es decir, "tener todo de acuerdo a una misma idea" no ha sido el resultado de una directiva predeterminada, sino de diálogo y decisión comunales. Como describió una líder comunitaria:

Siempre pensamos y actuamos en común. Incluso si tenemos pensamientos diferentes, y tal vez incluso discutir, al final terminamos uniéndonos porque es una fuerza. Lo que siempre hemos dicho: la fuerza es colectiva. (Fragmento entrevista grupal, 2014).

Como veremos en esta multitud de reflexiones desde la Comunidad, se hace hincapié en que la creación de una alternativa a la guerra requiere organización, compromiso y trabajo colectivo.
Terminé la transcripción de la entrevista señalada al inicio de este apartado, con la pregunta que le planteé sobre el 'camino' hacia la paz. Ella respondió con una narración sobre su viaje migratorio a San José desde el sur, y cómo se fundó la Comunidad de la Paz. Sin embargo, incluí mi pregunta en el fragmento porque refleja dónde estaba mi propia conceptualización de la paz en ese momento. Para mí, la paz aún era algo 'allá afuera', algo para alcanzar, para la cual había un sendero o caminos. Cuando volví a analizar el contenido de esta entrevista, percibí algo más. Ella comenzó su definición diciendo que la paz era 'tener' sin completar la frase. Cuando finalmente articuló su visión, cambió al 'estar': "estar todos juntitos". El primero sugiere la paz como una condición, el punto en el cual usted tiene algo. Este último, en cambio, postula la paz como una acción. Incluso en el momento de la entrevista, me impresionó la idea de que la paz no era algo que se podía esperar sino que había que crearla ahora.

\section{Crítica a la paz moderna}

Esta visión de paz como comunidad, en vez de una condición lograda a través de tratados estatales, se ve reflejada, además, en su crítica al 'proceso de paz' formal. La cita siguiente expresa un sentimiento común dentro de la comunidad, el que ellos no comparten el concepto de paz del Estado y lo que este busca a través de las negociaciones con las FARC:

La paz que habla Santos [...] es que la guerrilla no vaya a arremeter en contra de las fuerzas públicas. [...] En cambio, la fuerza pública sí puede, de una vez, tirarle a la guerrilla si los ven por ahí. [...] Dicen que la paz la están firmando, y cree que la paz nada más es no tener arremetidas en contra de la fuerza pública. [...] Pero seguro que las muertes no van a faltar [...] en la gente civil. Entonces, la paz que habla el gobierno [...] es que nadie tenga quejas sobre la fuerza pública. [...] [Que] el pueblo no diga nada. [...] Pero eso legalmente es un engaño. (Fragmento entrevista personal, 2014).

6 El enfoque de este artículo en San José de Apartadó, me impide hacer un análisis de los diversos imaginarios de paz en Colombia en medio de la coyuntura del "proceso de paz", tema que exploro en otro texto (de próxima publicación). 
Además, durante un taller realizado en la Universidad Campesina sobre el 'proceso de paz', otro miembro concordó: "La gente cree que en Colombia vamos para la paz. Pero para la paz de los cementerios" (Apuntes diario de campo, 2013). Otra miembro, con una perspectiva paralela a la 'paz negativa', opinó que la paz:

Es que no haya grupos armados. Para la Comunidad de Paz, la paz sería y es exigir un respeto de la vida y la tierra. Pero no creo en la paz. Seguirá el Estado, que igual es un grupo armado, con sus negocios, el narcotráfico. (Fragmento entrevista personal, 2014).

Estos miembros afirmaron que la desmovilización de las guerrillas no necesariamente pondrá fin a los ataques estatales en contra la protesta, aun si proviene de resistencias no violentas. Es decir, la paz requiere no sólo la desmovilización de la guerrilla y los paramilitares sino también del propio Estado (Gómez, 2015). La paz del Gobierno colombiano en vez de conllevar a la dignidad, según estos campesinos, equivale a una paz moderna de represión violenta de la disidencia contra la injusticia y la victoria de un grupo armado sobre otro (Foucault, 2003; Ross, 2011).

Otra voz escéptica sobre la posibilidad de la paz en este mundo, vino de un miembro profundamente religioso. Afirmó:

La paz del hombre es diferente a la paz de Dios. [...] La paz es lo que Jesús Cristo da al final, hay [solamente] una paz pasajera aquí. La Comunidad de Paz es una paz, pero no es verdadera, es momentánea. [...] Estamos aquí vivos. No estamos con la guerra, ni [...] con el Estado, con gente armada. [...] [La paz es] que haya comida. (Fragmento entrevista personal, 2014).

Según esta visión, la única paz posible surge de lo divino o de las formas de resistencia que aseguren la seguridad alimentaria. La concepción de otra miembro coincidió en que: "La paz es tranquilidad, pero por momentos. La Comunidad construye una paz. Gracias a la Comunidad, estamos medio tranquilos" (Fragmento entrevista personal, 2013). Su referencia al trabajo de la organización fue un tema común entre los miembros de la Comunidad de Paz. Significativamente, en las narrativas de los integrantes de la Comunidad, incluso cuando se define la paz como la tranquilidad o la ausencia de guerra, casi siempre se une esa concepción con afirmaciones de que tal tranquilidad, está constituida por el trabajo colectivo. Por ejemplo, para otra miembro, la paz es "Que no haya nada, nada aquí”, refiriéndose a los combates y la violencia. Ella continuó:

Que no hagamos ningún daño a los demás [...] que no haya armas ni nada. (Los miembros de la Comunidad de Paz) están trabajando fuertemente. Resistiendo, hombres y mujeres [...] para que haya la paz. Sin la paz no viviremos. Entonces, Dios propone que vivamos alegremente y nos ayudemos. (Fragmento entrevista personal, 2014).

Una vez más, se hace una conexión entre la paz y lo divino, y una correlación entre la paz y la vida.

Otro miembro de la Comunidad de Paz hizo una afirmación similar: La paz es "que no haya violencia. [...] Y un respeto a la vida. Que todos podamos vivir" (Fragmento entrevista personal, 2013). Insistir en que la paz es vida, refleja la posición racializada de la cual la Comunidad de Paz actúa y habla. En lugar de hablar desde la perspectiva de la sociedad civil, es decir, de cómo se puede fortalecer o 'reconstruir' la sociedad colombiana, ellos hablan desde una posición de exclusión y nonbeing (Fanon, 2008). La paz como la vida se define en oposición a la guerra colonial-moderna que los desplaza y asesina. La paz como la vida depende, por tanto, del fin de la guerra del Estado, pero este grupo de miembros descarta cualquier esperanza utópica de poner fin a esa guerra.

La Comunidad de Paz está siendo atacada precisamente porque representa una 'amenaza' para el Estado, como una alternativa política, pero también están siendo amenazados por el Estado (Goldberg, 2009) a causa de denunciar la 'supuesta' autoridad del Estado para legítimamente hacer la guerra contra sus súbditos (Finn, 2013). Para 
resumir, los sentidos acerca de la paz de este grupo de miembros de la Comunidad están alrededor del respeto por la vida del campesino. Pero ellos ven esa paz como una imposibilidad en la coyuntura actual. Por supuesto, esta perspectiva puede ser parcialmente explicada por la perspectiva cristiana de la que hablan muchos de estos miembros, en la que vivir en el mundo significa una lucha continua y sólo habrá paz a través de Dios en la próxima vida, conforme con las "grandes paces morales” según la clasificación de Dietrich (2012). No obstante, sostengo que esto también representa una denuncia del mundo moderno y sus conceptos de paz, tanto de la 'paz negativa' que parece ser inalcanzable, como de la 'paz positiva' enraizada en una 'sociedad civil' que por naturaleza excluye a estos campesinos de descendencia indígena. Es como si ellos dijeran: ' No hay paz en este sistema para nosotros!.

\section{Paz alternativa desde el trabajo comunitario, la solidaridad y la dignidad ecológica}

Sin embargo, la Comunidad no se limita a criticar esa paz liberal hegemónica. Traigo las voces anteriores para reflejar la diversidad de ideas dentro de la Comunidad, cuyos integrantes colectivamente, con sus diferencias, aún se comprometen a caminar juntos. Pero la mayoría de los miembros divergen de las perspectivas anteriormente citadas. Al contrario, tanto en el nombramiento de su organización en términos de la paz, como en las concepciones de paz de los demás integrantes, la paz no es un concepto rechazado, sino un concepto utilizado estratégicamente, practicado y reinventado más allá de las interpretaciones morales y modernas dominantes. Por ello en adelante me dedicaré a explorar esas visiones de una paz 'otra'.

Cuando se les pregunta sobre la paz, los miembros de la Comunidad tienden a responder aludiendo a los principios y los compromisos de su organización. Por ejemplo, refiriéndose a su regla de no colaborar directa o indirectamente con cualquier actor armado, una miembro dijo que la paz: "Es tener el poder de no ser parte de la guerra, de no colaborarle a ninguno de los actores. Es poder decidir de no ser parte" (Fragmento entrevista personal, 2013). Aquí, la paz se describe desde el lente del poder. Por lo tanto, la paz no es sólo rehusar la participación en la guerra, sino un rechazo a través del cual se instala un poder emancipador de autodeterminación, como el único medio para una alternativa dentro de las relaciones de poder moderno-colonial en torno a la dominación y la dependencia.

Otros miembros insistieron en que la paz no se crea simplemente por la negación de participar en la guerra, sino también por el trabajo colectivo activo. Un miembro inmediatamente hizo referencia a ambos: "A esa tropa armada ni [estar] con una ni con la otra. Eso [es] una parte. Ahora, otra cosa es de trabajar en grupo" (Fragmento entrevista personal, 2013). Este segundo componente se refiere específicamente a la regla comunitaria de pertenecer a un grupo de trabajo agrícola y alude al trabajo del Comunitario semanal. Otras citas expresan lo mismo:

[La paz] es todos juntos. Imagínese, cuando hay un trabajo salen todos! ¡Y siempre! (Fragmento entrevista personal, 2013). Trabajando bien unidos. Y un respeto, del uno al otro. Es respeto, ¡amor! (Apuntes diario de campo, 2014). La paz es que estemos unidos todos. Porque todos somos hijos de Dios. Seamos blancos o negros, todos somos hijos de Dios. Porque unidos podemos trabajar. (Fragmento entrevista personal, 2013).

En la última, el único miembro que aludió al racismo, reitera un principio central de la Comunidad de Paz, el de la pluralidad y el anti-racismo (Comunidad de Paz de San José de Apartadó, 2005). Una vez más, se enfatiza en que la solidaridad, el respeto, el amor y la unidad comunitaria hacen posible una paz alternativa. Así, la paz se define no como una tranquilidad o descanso del trabajo, sino como el trabajo en comunidad mismo. Es más, un miembro, cuando se le preguntó qué significaba para él la paz, respondió a partir de la noción de la resistencia: "La resistencia es buena, porque la Comunidad de Paz defiende [...] para hacer un mundo más distinto, sin hambre, sin 
muertos. No como esa gente, un poder capitalista" (Fragmento entrevista personal, 2013). Por consiguiente, la paz es explícitamente una cuestión de crear alternativas al capitalismo. Podríamos decir que tal poder radical de resistir el desplazamiento y de negarse a participar en la guerra depende de, y se construye a través de, una política de paz transrelacional construida desde el compromiso y la solidaridad, la cual rompe con la violencia reaccionaria. Cabe anotar nuevamente que no se trata de una condición sino un proceso de acciones que crean otro sujeto político. Se puede observar en la siguiente definición cómo el miembro citó dos pilares organizativos - el trabajo comunitario y la negación de participación en la guerra-pero también la meta de transformarse en 'personas de paz'

La paz para mí significa [...] uno, no vivir en la guerra, no ser parte de la guerra, no apoyar al Estado, sino vivir en comunidad. Buscar la paz [...] dialogando. [...] La verdad [es que] quiero ser, al menos, un hombre de paz. [...] Las armas para mí no me gustan, solamente las herramientas para trabajar. (Fragmento entrevista personal, 2013).

Esta concepción refleja una 'No violencia de principio' más allá de una no violencia pragmática (Sharp, 1973). Rechaza el uso de las armas por principio, y no meramente como una decisión estratégica. Es un ser político que no se reduce a alcanzar ciertas condiciones materiales, sino que busca crear y cultivar un sujeto de paz. Otro integrante describió su transformación subjetiva desde que se unió a la Comunidad de Paz:

[...] Antes de entrar a la Comunidad [...] la vida mía era otro, ¡otro mundo! El mundo mío era seguir esa ideología capitalista. [.... La paz no es hablar. Sino es vivirla. (Fragmento entrevista personal, 2014).

La paz como algo que hay que vivir en la cotidianidad comunitaria fue una idea promovida por el líder fallecido Eduar Lanchero. Según citó el padre Javier Giraldo (Fragmento entrevista personal, 2013), en la peregrinación de la Comunidad de Paz a Sumapaz en el 2010, Lanchero argumentó que, "La paz no es de mañana ¡sino de hoy! Ahora hay paz porque hay comunidad". Otro miembro narró, citando a Lanchero, que:

La paz sería, ser solidarios, el uno con los otros. [...] Es hacer comunidad. [...] Lo que decía Eduar, la paz la podemos hacer nosotros. No es que esperemos a los grupos [armados, o] que haya la paz desde la Habana. (Apuntes diario de campo, 2014).

Sin embargo, la idea de que la propia comunidad puede hacer y ser la paz en medio de la guerra es una idea disputada. Por ejemplo, consideremos el siguiente intercambio durante un taller de la Universidad Campesina sobre tenencia de la tierra en 2013:

Un líder de la Comunidad de Paz declaró que su estrategia era "Seguir trabajando, y con otras comunidades. Nosotros, ya somos ejemplo de la paz, que sí es posible la paz”. Una ex-acompañante internacional respondió, "Pero, perdóneme, ¿qué paz están viviendo ustedes acá con los actores armados rodeándolos?" El líder contestó: "No vamos a esperar que los actores [armados] se pongan de acuerdo. [...] Vamos a ser un ejemplo de paz si nos respetan o no nos respetan, que sí es posible. Si la espera de ellos, es un problema. Día a día, no se necesita que los actores armados se pongan de acuerdo. (Apuntes diario de campo, 2013).

La Comunidad de Paz se describe frecuentemente como un ejemplo o experiencia de paz, pero no como un 'modelo' de paz. No recomiendan que se replique su estrategia del mismo modo en otros lugares, sino que esperan que la Comunidad sirva de inspiración para que otras personas creen sus propias comunidades en resistencia. Es un llamado a la imaginación para crear y ser paz inmediatamente, en nuestros propios términos. Por supuesto, la acompañante también tenía razón: la guerra estatal-paramilitar continúa. Una miembro, de hecho, al hablar de paz, argumentó, "Eduar decía: van a retornar a [las veredas], pero no es que no venga a haber golpes. Habrá golpes en los alrededores" (Fragmento entrevista personal, 2013). Por consiguiente, esto no es un imaginario utópico. Se reconoce que la paz requiere lucha, provocando ataques de represalia por parte de los actores armados. 
Sin embargo, tal violencia no elimina la realidad de la paz como comunidad aquí y ahora. En las palabras de Lanchero, en un documental sobre la Comunidad de Paz "[...] la lógica del sistema de muerte, de los paramilitares, del Estado, es desplazar al pueblo. Quieren aterrorizar a la gente porque genera un individualismo tan fuerte para que ya no le importe la vida del otro" (Buenaventura, 2011). Por lo tanto, la creación de otros mundos requiere una alternativa a la guerra y al capitalismo que individualizan a los sujetos (Foucault, 1982) y socavan la resistencia (Lanchero, 2002). Lanchero continua:

Es por eso que la única manera era vivir en una comunidad. Si vives en una comunidad tienes diferentes maneras de relacionarse, de organizar, de participar, de solidaridad donde todos se preocupan por la vida del otro. (Buenaventura, 2011).

En otras palabras, la paz no se construye individualmente. Para citar a la acompañante internacional Michaela Soellinger, "La paz es relacional" (Fragmento entrevista personal, 2013), creada a través del afecto-cuidado para la vida (Gómez Correal, 2016). Requiere colectividad, solidaridad y organización.

Esta paz no se queda en las palabras sino se construye a través de las prácticas cotidianas de resistencia campesina, como señaló un miembro: "Nosotros, la Comunidad de Paz, tenemos [...] el trabajo comunitario, que es todo. $\mathrm{Y}$ el trabajo en grupo. Esos son los compromisos" (Fragmento entrevista personal, 2012). Para re-presentar cómo se realiza el trabajo comunitario semanal, citaré a un acompañante internacional, quien describió la primera vez que lo acompañó:
Mi primer acompañamiento fue para un Comunitario dos días después de mi llegada. Estaban trabajando para arreglar el camino de San José a La Unión. Fueron unas 40 personas, niños, mujeres, un Comunitario donde todos estaban en el mismo equipo. [...] Yo estaba allí alrededor de las 7 de la mañana, y ya [cuatro hombres] estaban allí. En lo que era sólo un trozo de barro. [...] El trabajo mismo implicó, primero, mover todas las rocas grandes fuera del camino de donde iba a ser [...] [y después] ir al río para recoger pequeñas piedras, colocando las rocas grandes abajo y colocando las pequeñas rocas abajo. Yendo al río, recogiendo sedimentos, como arena aluvial, y luego cubriendo las pequeñas rocas, y luego forrando el camino con rocas más grandes. ¡Y creo que la mayor parte de esto se hizo para el beneficio de los caballos! Eso es un bien público: la comunidad se reunió, y había niños, viejos, y ihabía un chico cuyo trabajo específico era perseguir avispas! Tenía un palo, y [un miembro] le echó gasolina y el niño estaba persiguiendo a las avispas [...] y ese era su trabajo específico. Eso fue justo después de que llegué aquí. Me impresionó. [...] No sólo la comunidad usa ese camino, [hasta] los guerrilleros [...] [y] los militares usan ese camino. [...] era un bien público, y beneficiaba a todos. [...] Había como 40 personas, [cada una haciendo] unas 12 horas de trabajo. $\mathrm{Y}$ eso fue solamente ese día. La semana siguiente lo hicieron para otras partes. (Fragmento entrevista personal, 2013) (Relato original en inglés) ${ }^{7}$.

El trabajo colectivo no se limita al Comunitario semanal y los grupos de trabajo. La Comunidad de Paz también realiza peregrinaciones para conmemorar a sus víctimas. Por ejemplo, cada febrero, la comunidad y representantes de otras organizaciones solidarias congregan en el sitio de la masacre del 2005 en las veredas de Mulatos y La Resbaloza, donde ocho personas, incluyendo tres menores

7 Texto original en ingles "My first accompaniment was for a comunitario two days after I arrived. They were working on improving the path from San José to La Unión. It was about 40 people, kids, women, a comunitario where everyone was on the same team. [...] I was there around 7am, and already [four men] were there. On what was just a patch of mud. [...] The actual work involved first moving all the big rocks out of the way of the path where it was going to be [...] [then] going to the river collecting small rocks, laying the big rocks down, laying the small rocks down, going to the river, collecting silt, like alluvial sand, and then covering the small rocks, and then lining the camino with larger rocks. And I think most of this was done for the benefit of horses!!! That is a public good: the community came together, and there were children, old people, and there was one kid whose specific job was to chase wasps away!!! He had a palo, and [one man] poured gasoline on top and the kid was chasing wasps away [...] and that was his specific job. That was right after I first got here. It made an impression. [...] Not only the community uses that road, [even] guerrillas [...] [and] the military use that road. But it was a public good, and it benefitted everyone. [...] There were like 40 people, [each doing] about 12 hours worth of work. And that was just that day. The next week they did it for other parts".

Polisemia No. 22, 55 - 72. Comunidad de Paz: Una paz 'otra' en San José de Apartadó-Colombia. Bogotá, ISSN: 1900-4648. Julio - diciembre de 2016 
de edad y el fundador comunitario Luis Eduardo Guerra, fueron asesinados con machetes en una operación militar-paramilitar. La Comunidad también conmemora los campesinos asesinados haciendo movimientos de víctimas con piedras pintadas con los nombres de las personas. En medio de la impunidad imperante en Colombia, esta memoria es una manera de hacer una justicia propia, a través de la cual se fortalece el tejido y el compromiso comunitarios. Una de las integrantes, Doña Brígida González, describe la fuerza que se deriva de la memoria, que incluye el mantener contacto y la relacionalidad con el espíritu de los muertos:

La memoria es la fuerza de la resistencia. [...] Nosotros mantenemos la memoria y siempre sentimos que alguien que faltó, de esa gente tan importante que ha faltado en la Comunidad, que siguen estando con nosotros. Entonces es como que no se fueron. Siguen estando, desde el más allá. Está su espíritu con nosotros. Y yo creo que ese ha sido la característica de nuestra Comunidad, y es lo que nos da esa fuerza. (Fragmento entrevista personal, 2011).

Estas prácticas de memoria nutren una relacionalidad entre los vivos y los muertos, en la que hay paz como dignidad y solidaridad no sólo entre los vivos sino también con los muertos. Aquí, la muerte no es un fin sino un comienzo político (Gómez Correal, 2016) de una "otra política" emancipadora de autodeterminación y dignidad (Courtheyn, 2016b).

Además, la paz relacional de la Comunidad de Paz también se construye con la biosfera. Doña Brígida González insistió en que se ampliara el análisis de la guerra colombiana más allá de sus efectos sobre los seres humanos, para reconocer que la guerra es también en contra de la naturaleza, la cual perjudica la vida de la tierra, el agua y todos los seres vivos:

Estamos haciendo la guerra en todos los sentidos. Con la naturaleza. [...] [Se mata] a una persona y ya es como matar a cualquier animal. [Los] animales también tienen derecho a vivir. Pero como seres humanos yo creo que [...] el valor a la vida se ha perdido de una forma impresionante. [...] Sin la tierra, nosotros no pudiéramos vivir. [...] La Madre Tierra nos tiene de todo. Nos tiene el agua que es vida. Tiene las plantas que son la alimentación diaria de nosotros. (Fragmento entrevista personal, 2013).

Esta insistencia, en torno a la armonía entre todos los seres parte de un sentido relacional con la naturaleza y lo divino, el cual que refleja una 'paz energética' (Dietrich 2012). Para contrarrestar la degradación de la biósfera, la Comunidad de Paz prohíbe la explotación minera en sus espacios. Por ejemplo, una líder explicó esa política afirmando que la tierra también es un ser vivo que se necesita cuidar:

Nosotros no somos exploradores ni vamos a explotar minas. [...] La tierra [...] yo [la] comparo mucho con el cuerpo del ser humano [...] Porque se le quita minerales. Los tiene porque [...] los necesita. Pues si le quita todo eso, le estamos quitando vida. A la tierra. Como dicen los indígenas, la Madre Tierra. (Fragmento entrevista grupal, 2014).

Otro líder reiteró lo antagónico de la relación que ellos sostienen con la tierra como comunidad y la de los agentes que representan los intereses estatales-empresariales en San José de Apartadó:

La tierra es nuestra madre. De ella fuimos tomados. Fuimos hechos del polvo de la tierra. Y ella nos da nuestra alimentación. [...] Estas son tierras riquísimas. Y por eso están peleando esta tierra y nos quieren sacar $[\ldots]$ por la riqueza que tenemos. Especialmente, un aire puro [y] agua pura. Y que la Serranía de Abibe es muy rica $[. .$.$] en lo que tiene que ver con el carbón,$ muchos minerales, oro, etcétera. Y están entregadas ya a una multinacional. (Fragmento del documental: Moncada, Cristancho \& Caro, 2011).

Al pensar la tierra como un ser vivo, otro miembro de la Comunidad, explicó su oposición al monocultivo:

El problema es que el campesino quiere que el cultivo sea de una cosa, y ya. Pero yo les voy diciendo, si una persona va comiendo banana solamente, le hace mal a la salud. Pues, para la tierra es lo mismo. Hay que sembrar muchas cosas y eso va alimentando la tierra. (Apuntes diario de campo, 2013). 
Estos miembros de la Comunidad expresan una relación comunicativa con la tierra. La tierra no es simplemente un objeto inerte, sino un ser vivo que requiere alimento y una 'dieta variada y saludable', al igual que los seres humanos. Sin utilizar el lenguaje de los "seres de la tierra" (De la Cadena, 2015) o practicar rituales espirituales para conectarse con la Madre Tierra (Vachon, 2011), la comprensión de estos campesinos sobre la tierra es similar a muchos grupos indígenas en las Américas.

Además, la cita señala cómo la agricultura en pequeña escala no es necesariamente 'sostenible': una paz relacional es el producto de la práctica política, no una 'esencia' de ciertos pueblos. Al insistir en la paz no solamente para los seres humanos sino también para la tierra y los animales, ellos describen lo que clasifico como la dignidad ecológica: el equilibrio de la vida de todos los seres de la biosfera, la cual presenta una medida para lidiar con la crisis ecológica en la actualidad.

La Comunidad de Paz busca la dignidad ecológica fundando centros agrícolas y granjas autosuficientes, en las que prohíben la exploración minera, preservan semillas y plantas medicinales, y experimentan con la soberanía alimentaria. El coordinador de un centro agrícola narra los desafíos y los éxitos del proyecto comunitario de producción de panela, afirmando la importancia de la autosuficiencia:

Me da tanta felicidad de sacar la miel de caña. Ha habido muchas dificultades. La gente decía, "que no, muy difícil. No van a sacar miel ni panela”. Pero algunos cuchitos me decían, ¡sí! ¡Esto sirve! He trabajado la caña, cuando era un niño. Los indígenas vinieron aquí a mostrarnos a hacer panela. Y arrancamos. La primera, se perdió. [...] [A] mucha gente hay que mostrarles que sí las cosas son posibles. Que vean porque no creen. "No la tomo yo, no". Y las personas ya están felices, sacando panela. Tiene un buen sabor. [...] Para las comunidades es importante no depender de afuera, no depender del dinero. Entonces estamos muy atrasados, pero ihay que trabajar muy duro! Primero es la autosuficiencia en alimentación. [...] Me alegra mucho cuando gente me dice, "tengo un lago de peces y allí lo aprendí en el Centro Agrícola". Esto me da mucha fuerza para seguir, buscando más alternativas. (Fragmento entrevista personal, 2012).

La producción de panela en ese centro agrícola ya provee para el consumo de la mayoría de los habitantes de esa vereda (Apuntes diario de campo, 2014). Es decir, la paz como dignidad ecológica requiere la construcción de soberanía alimentaria y el trabajo colectivo desde las comunidades. Así como, esas iniciativas ayudan a los campesinos a percibir la posibilidad de alternativas, la Comunidad de Paz en sí ilumina la posibilidad de una paz alternativa.

En última instancia, sin embargo, esta praxis de la paz está intrínsecamente antagónica con la actual 'acumulación por desposesión' y la dependencia impuesta del sistema económico capitalista (Harvey, 2005) y el neoextractivismo (Dávalos, 2011; Márquez Mina, 2015). De hecho, al definir la paz, varios miembros de la Comunidad insistieron en el rechazo del extractivismo y de la dependencia, proponiendo la autonomía a través de la soberanía alimentaria. Por ejemplo:

El Estado quiere mandar en todo, no quiere que nadie le supere, sino quiere tenerlos ahí. [...] [Queremos] no depender del papá Estado. [Si se dice] "que nos manden esto" [...] uno termina esperando. [...] Somos autónomos. Nosotros queremos tener nuestros propios cultivos, la alimentación, nuestra propia educación. Y la tenemos. Las alternativas de vivir. [...] Hay una paz en nuestro interior, en nuestro entorno. [...] Si nosotros nos unimos, luchamos, sembramos, trabajamos y tenemos el pancoger diario, ahí va a haber una paz. Una paz, aunque, atacados por el Estado. (Fragmento entrevista personal, 2014).

En otras palabras, la paz como comunidad se produce a través de la autonomía política, la soberanía alimentaria, la educación autónoma, la convivencia y la solidaridad. Cabe anotar que en lugar de una 'zona humanitaria', en la cual podría haber una 'paz' temporal o espacial de refugio, el acto de organizarse como una comunidad de paz exige un mayor grado de unidad, solidaridad y trabajo colectivo entre el grupo. 
Sin embargo, estas prácticas no se hacen de manera aislada sino a través de redes. La Comunidad de Paz es protagonista en redes nacionales e internacionales de derechos humanos, además de redes campesinas como la Universidad Campesina de Resistencia, en la cual campesinos indígenas y afrodescendientes se encuentran puntualmente para compartir y generar conocimiento en talleres sobre las prácticas agrícolas, las plantas medicinales, las comunicaciones y los derechos, talleres que, por ejemplo, contribuyeron al proyecto de panela en el centro agrícola anteriormente mencionado.

Este tipo de paz es un intento de ruptura con la guerra colonial moderna-colonial-neoliberal (Reyes, 2015). Pero no es una paz de resignación o parroquialismo. La paz para la Comunidad de Paz consiste en, primero, resistir a la guerra negándose a colaborarles a los actores armados estatales y no estatales, y segundo, creando dignidad ecológica a través de la relacionalidad, la solidaridad y el trabajo comunitario dentro de la Comunidad y con otras comunidades en resistencia.

\section{Conclusión}

Como un aporte a los estudios de paz y la exploración creciente de una multitud de diferentes ideas y prácticas de la paz alrededor del mundo, este artículo ilumina la praxis de una paz campesina en la emblemática Comunidad de Paz de San José de Apartadó, Colombia. En lugar de depender de lo que deberían hacer los Estados y grupos armados, esta experiencia encarna una perspectiva activa y empoderada de la paz, centrándose en lo que las comunidades pueden hacer inmediatamente si se organizan colectivamente. A partir de colaboraciones con otras comunidades campesinas que conjuntamente luchan por la autonomía y la vida, se realiza lo que he denominado como la paz trasrelacional radical: la dignidad ecológica creada en redes solidarias de comunidades en resistencia. La paz carece de sentido si no representa procesos transformadores de ruptura, que contrarresten la crisis ecológica de la modernidad arraigada en la violencia interseccional de los dualismos y la destrucción de la biósfera. A su vez, articulo la paz trasrelacional radical como un marco conceptual para evaluar las many peaces iluminadas por las nuevas geografías de paz y las investigaciones sobre trans-rational peace, de manera que hay paz sólo en la medida en que se construye relacionalidad y vida digna.

\section{Referencias}

Buenaventura,R.(2011).Hopefor Colombia: The Grace Pilgrimage 2010 Bogotá. Portugal: Grace Media Productions. Recuperado de http://www.youtube.com/watch?v=8vhh5KNnrxA\&list=UUe823wgIRspP8paQif-6Vg\&index $=15$

Ceceña, A. E. (2012). On the Complex Relation between Knowledges and Emancipations. South Atlantic Quarterly, 111(1), 111-132.

Comunidad de Paz de San José de Apartadó. (2005). Nuestros Principios en la Comunidad de Paz en San José de Apartadó. En Noche y Niebla. Caso Tipo No. 6. Comunidad de Paz de San José de Apartadó (pp. 13-15). Bogotá: Centro de Investigación y Educación Popular (Cinep).

Courtheyn, C. (2016a). Memory is the strength of our resistance: A critical performance geography of peace, memory, territory, and politics in the San José Peace Community, Colombia [Tesis de doctorado]. University of North Carolina at Chapel Hill, ProQuest Dissertations Publishing.

Courtheyn, C. (2016b). Memory is the strength of our resistance: an "other politics" through embodied and material commemoration in the San José Peace Community, Colombia. Social \& Cultural Geography, 17(7), 933-958.

Dalby, S. (2014). Peace and critical geopolitics. En F. McConnell, N. Megoran, y P. Williams (eds.), Geographies of Peace (pp. 29-46). New York: I.B. Tauris.

Daley, P. (2014). Unearthing the local: hegemony and peace discourses in Central Africa. En F. McConnell, N. Megoran, y P. Williams (eds.), Geographies of Peace (pp. 66-86). New York: I.B. Tauris.

Darling, J. (2014). Welcome to Sheffield: the lessthan-violent geographies of urban asylum. En F. McConnell, N. Megoran, y P. Williams 
(eds.), Geographies of Peace (pp. 229-249). New York: I. B. Tauris.

Dávalos, P. (2011). La democracia disciplinaria: el proyecto posneoliberal para América Latina. Bogotá: Ediciones desde abajo.

De la Cadena, M. (2000). Indigenous Mestizos: The Politics of Race and Culture in Cuzco, Peru, 1919-1991. Durham: Duke University Press.

De la Cadena, M. (2015). Earth Beings: Ecologies of practice across Andean worlds. Durham: Duke University Press.

Denis, R. (2012). The Birth of an "Other Politics" in Venezuela. South Atlantic Quarterly, 111(1), 81-93.

Dietrich, W. (2002). Farewell to the One Peace. Peace Review. A Journal of Social Justice, 14 (1), 49-55.

Dietrich, W. (2011). Beyond the Gates of Eden: Transrational Peaces. En W. Dietrich, J. Echavarría, G. Esteva, D. Ingruber y N. Koppensteiner (eds.), The Palgrave International Handbook of Peace Studies: A Cultural Perspective (pp. 3-23). New York: Palgrave Macmillan.

Dietrich, W. (2012). Interpretations of Peace in History and Culture. New York: Palgrave Macmillan.

Dietrich, W. (2014). A Brief Introduction to Transrational Peace Research and Elicitive Conflict Transformation. Journal of Conflictology, 5(2), 48-57.

Dietrich, W., Echavarría J., Esteva, G., Ingruber, D., \& Koppensteiner, N. (eds.). (2011). The Palgrave International Handbook of Peace Studies: A Cultural Perspective. New York: Palgrave Macmillan.

Dussel, E. (2000). Europe, Modernity, and Eurocentrism. Neplanta: Views from South, 1(3), 465-78.

Escobar, A. (s.f). Designs for the Pluriverse. Durham: Duke University Press.

Escobar, A. (2014). Sentipensar con la tierra: Nuevas lecturas sobre desarrollo, territorio y diferencia. Medellín: Ediciones unaula.

Fanon, F. (2008). Black Skin, White Masks. New York: Grove Press.

Fasheh, M. (2011). Shalom/Salaam: A Personal
Palestinian Perspective. En W. Dietrich, J. Echavarría, G. Esteva, D. Ingruber y N. Koppensteiner (eds.), The Palgrave International Handbook of Peace Studies: A Cultural Perspective (pp. 99-120). New York: Palgrave Macmillan.

Federici, S. (2010). Feminism and the Politics of the Commons in the Era of Primitive Accumulation. En Team Colors Collective (ed.), Uses of a Whirlwind: Movement, Movements, and Contemporary Radical Currents in the United States (pp. 283-293). Oakland: AK Press.

Finn, L. (2013). The State and the Comunidad de Paz. All Quiet on the Hillside. Recuperado de https://lukefinn.wordpress.com/2013/09/18/ the-state-and-the-comunidad-de-paz/

Foucault, M. (1982). The Subject and Power. Critical Inquiry, 8 (4), 777-795.

Foucault, M. (1984). What Is Enlightenment? En P. Rabinow, (ed.), The Foucault Reader (pp. 32-50). New York: Pantheon Books.

Foucault, M. (2003). "Society Must Be Defended" Lectures at the College de France 1975-76. New York: Picador.

Galtung, J. (1964). What is peace research? Journal of Peace Research, 1(1), 1-4.

Galtung, J. (1996). Peace by Peaceful Means: Peace and Conflict, Development and Civilization. Oslo \& Thousand Oaks, CA: International Peace Research Institute \& Sage Publications.

Gebrewold, B. (2011). T’ùmmu: An East African Perspective. En W. Dietrich, J. Echavarría, G. Esteva, D. Ingruber y N. Koppensteiner (eds.), The Palgrave International Handbook of Peace Studies: A Cultural Perspective (pp. 428-441). New York: Palgrave Macmillan.

Giraldo, J. (2010). Fusil o Toga, Toga y Fusil: El Estado Contra la Comunidad de Paz de San José de Apartadó. Bogotá: Editorial Códice.

Goldberg, D. T. (2009). The Threat of Race: Reflections on Racial Neoliberalism. Malden, MA: Wiley-Blackwell.

Gómez, D. M. (2015). Entre la movilización y sus desenlaces: la transformación emancipatoria, los movimientos de derechos humanos 
y de víctimas en Colombia. En R. Romero y O. Solis (eds.), Resistencias locales, utopias globales (pp. 85-106). México, D.F.: Sindicato de Trabajadores de la Universidad Nacional Autónoma de México.

Gómez, D. M. (2016). De amor, vientre y sangre: Politización de lazos íntimos de pertenencia y cuidado en Colombia. Otras Palabras, 23, 103-119.

Grupo Acontecimiento. (2012). The Affirmation of an Other Politics of Emancipation. South Atlantic Quarterly, 111(1), 29-49.

Grupo de Memoria Histórica. (2013). ¡BASTA YA! Colombia: Memorias de guerra y dignidad. Bogotá: Imprenta Nacional.

Gutiérrez, R. (2012). The Rhythms of the Pachakuti: Brief Reflections Regarding How We Have Come to Know Emancipatory Struggles and the Significance of the Term Social Emancipation. South Atlantic Quarterly, 111(1), 51-64.

Harvey, D. (2005). The New Imperialism. Oxford: Oxford University Press.

Jacobsen, E. K. U. (2011). Friđr: A Northern European Perspective. En W. Dietrich, J. Echavarría, G. Esteva, D. Ingruber y N. Koppensteiner (eds.), The Palgrave International Handbook of Peace Studies: $A$ Cultural Perspective (pp. 67-86). New York: Palgrave Macmillan.

Koopman, S. (2011a). Alter-geopolitics: Other securities are happening. Geoforum, 42(3), 274-284.

Koopman, S. (2011b). Let's take peace to pieces. Political Geography, 30(4), 193-194.

Lanchero, E. (2002). El amanecer de las resistencias. Bogotá: Editorial Códice.

Loyd, J. M. (2012). Geographies of Peace and Antiviolence. Geography Compass, 6(8),477-489.

Madison, D. S. (2010). Acts of Activism: Human Rights as Radical Performance. Cambridge: Cambridge University Press.

Madison, D. S. (2012). Critical Ethnography: Method, Ethics, and Performance (2a ed.). Los Angeles: Sage Publications.
Mahony, L., \& Escobar, L. E. (1997). Unarmed bodyguards: International accompaniment for the protection of human rights. West Hartford, Conn.: Kumarian Press.

Márquez, F. (2015). La conmovedora carta de Francia Elena Márquez Mina líder afro del Cauca, un llamado a la reflexión nacional. Radio Macondo. Recuperado de http://radiomacondo.fm/2015/04/22/la-conmovedora-cartade-francia-elena-marquez-mina-lider-afro-delcauca-un-llamado-a-la-reflexion-nacional/

Martínez Hincapié, C. E. (2010). Paradigmas de transformación social y Noviolencia. En C. E. Martínez Hincapié, J. A. Espejo Ramírez, \& Ó. Useche Aldana (eds.), Noviolencia: Creando mundos posibles (pp. 15-38). Bogotá: UNIMINUTO.

Martínez, C. E. (2013). La cultura emergente de la no-violencia y la crisis de la civilización. Revista Vectores de Investigación, 7(7), 159-182.

McConnell, F. (2014). Contextualizing and politicizing peace: geographies of Tibetan satyagraha. En F. McConnell, N. Megoran, y P. Williams (eds.), Geographies of Peace (pp. 131150). New York: I.B. Tauris.

McConnell, F., Megoran, N., y Williams, P. (eds.). (2014). Geographies of Peace. New York: I. B. Tauris.

Megoran, N. (2011). War and peace? An agenda for peace research and practice in geography. Political Geography, 30(4), 178-189.

Megoran, N. (2014). "Migration and Peace: The Transnational Activities of Bukharan Jews." En F. McConnell, N. Megoran, y P. Williams (eds.) Geographies of Peace (pp. 212-28). New York: I.B. Tauris.

Megoran, N., Williams, P., \& McConnell, F. (2014). Geographies of peace, geographies for peace. En F. McConnell, N. Megoran, y P. Williams (eds.), Geographies of Peace (pp. 250 260). New York: I.B. Tauris.

Mitchell, C. (2012). Forward. En Interpretations of Peace in History and Culture (pp. vii-viii). New York: Palgrave Macmillan. 
Moncada Hurtado, C., Cristancho Ossa, F., y Caro Morales, A. M. (2011). Comunidad de Paz, resiste (parte 1). Colombia: Universidad Católica de Pereira. Recuperado de https:// www.youtube.com/watch? $\mathrm{v}=\mathrm{jkVDm} 4 \mathrm{wsr}$ b8 \&list $=$ PL 3cBDgCi9tR3D 1 zuCfZ3z2 $y G \_f N Q F N d f n \& i n d e x=1$

Muñoz, F. (2001). La paz imperfecta en un universo en conflicto. En J. Muñoz (ed.), La Paz Imperfecta (pp. 2-66). Granada: Universidad de Granada.

Pollock, D. (2005). Introduction: Remembering. En D. Pollock (ed.), Remembering: Oral History Performance (pp. 1-17). New York: Palgrave Macmillan.

Rengifo,V.G.(2011). Thaq: An Andean-Amazonian Perspective. En W. Dietrich, J. Echavarría, J. Esteva, D. Ingruber y N. Koppensteiner, (eds.). The Palgrave International Handbook of Peace Studies: A Cultural Perspective (pp. 373-386). New York: Palgrave Macmillan.

Reyes, Á. (2015). Zapatismo: other geographies circa "the end of the world." Environment and Planning D: Society and Space, 33(3), 408-424.

Rojas, C. (2002). Civilization and Violence: Regimes of Representation in NineteenthCentury Colombia. Minneapolis: University of Minnesota Press.

Ross, A. (2011). Geographies of war and the putative peace. Political Geography, 30(4), 197-199.

Rueda, E. A., Alvarado, S. V., y Gentili, P. (eds.). (2016). Paz en Colombia: Perspectivas, desafíos, opciones. Ciudad Autónoma de Buenos Aires: CLACSO.

Santos, B. (2002). Toward A New Legal Common Sense: Law, Globalization, and Emancipation (2a ed.). London: Butterworths LexisNexis.

Sharp, G. (1973). The Politics of Nonviolent Action. Boston: Porter Sargent.

Silva, D. F. (2012). "Terror-rialización y parrbe- sía: La emancipación social desde los procesos de organización campesina En Colombia" En D. F. Silva (ed.). Critica, Emancipación y Construcción de Paz (pp. 162-77). Bogotá: Corporación Universitaria Minuto de Dios -UNIMINUTO.

Steiner, C. (2000). Imaginación y poder: El encuentro del interior con la costa en Urabá, 1900-1960. Medellín: Editorial Universidad de Antioquia. Ulloa, A. (2012). Los territorios indígenas en Colombia: de escenarios de apropiación transnacional a territorialidades alternatives. Scripta Nova: Revista Electrónica de Geografía $y$ Ciencias Sociales, 16.

Useche Aldana, Ó. (2016). Ciudadanias en resistencia: El acontecimiento del poder ciudadano y la creación de formas noviolentas de re-existencia social. Bogotá: UNIMINUTO.

Vachon, R. (2011). Kayanerekowa: A Mohawk Perspective. En: W. Dietrich, J. Echavarría, J. Esteva, D. Ingruber, y N. Koppensteiner (eds.). The Palgrave International Handbook of Peace Studies: A Cultural Perspective (pp. 330-351). New York: Palgrave Macmillan.

Vasudevan, P. (2012). Performance and Proximity: Revisiting environmental justice in Warren County, North Carolina. Performance Research, 17(4), 18-26.

Wallerstein, I. (2004). World-Systems Analysis: An Introduction. Durham: Duke University Press.

Wilderson, F. B. (2010). Red, White \& Black: Cinema and the Structure of U.S. Antagonisms. Durham: Duke University Press.

Williams, P. (2013). Reproducing Everyday Peace in North India: Process, Politics, and Power. Annals of the Association of American Geographers, 103(1), 230-250. 